\title{
Expert consensus of perioperative intensive care and management of critically ill cancer patients (2021)
}

\author{
Haijun Wang', Hongzhi Wang', Wei Chen ${ }^{3}$, Heling Zhao ${ }^{4}$, Yuanyu Qian ${ }^{5}$, Limin Shen ${ }^{4}$, Shuangling Li ${ }^{6}$, \\ Jun Duan ${ }^{7}$, Zhiqiang Wang ${ }^{8}$, Keliang Cui ${ }^{9}$, Quan Wang ${ }^{10}$, Xiaoyan Xue ${ }^{11}$, Xiyuan Li ${ }^{12}$, Liwei Hua ${ }^{13}$, \\ Yingping Zhang ${ }^{14}$, Yongshun Feng ${ }^{15}$, Huaiwu $\mathrm{He}^{16}$, Lei $\mathrm{Li}^{17}$, Nan Zhang ${ }^{18}$, Jun Dong ${ }^{2}$, Weishuai Bian ${ }^{3}$, \\ Feiping $\mathrm{Lu}^{3}$, Donghao Wang" ${ }^{9 *}$ Yun Long ${ }^{16 \#}$, Xuezhong Xing ${ }^{1 \# \wedge}$; on behalf of Critical Care Medicine \\ Committee of Beijing Association of Oncology (CCMBAO)
}

\begin{abstract}
${ }^{1}$ Department of Intensive Care Unit, National Cancer Center/National Clinical Research Center for Cancer/Cancer Hospital, Chinese Academy of Medical Sciences and Peking Union Medical College, Beijing, China; ${ }^{2}$ Department of Intensive Care Unit, Peking University Cancer Hospital, Beijing, China; ${ }^{3}$ Department of Intensive Care Unit, Beijing Shijitan Hospital, Capital Medical University, Beijing, China; ${ }^{4}$ Department of Intensive Care Unit, Hebei General Hospital, Hebei, China; ${ }^{5}$ Department of Internal Medicine, Chinese PLA General Hospital, Beijing, China; ${ }^{6}$ Department of Intensive Care Unit, Peking University First Hospital, Beijing, China; ${ }^{7}$ Department of Intensive Care Unit, China-Japan Friendship Hospital, Beijing, China; ${ }^{8}$ Department of Intensive Care Unit, Tianjin Medical University General Hospital, Tianjin, China; ${ }^{9}$ Department of Intensive Care Unit, Tianjin Medical University Cancer Institute \& Hospital, Tianjin, China; ${ }^{10}$ Department of Intensive Care Unit, Beijing Children's Hospital, Capital Medical University, Beijing, China; ${ }^{11}$ Department of Intensive Care Unit, Aerospace Corporation Central Hospital of China, Beijing, China; ${ }^{12}$ Department of Intensive Care Unit, Civil Aviation General Hospital, Beijing, China; ${ }^{13}$ Department of Intensive Care Unit, Affiliated Hospital of Chengde Medical University, Chengde, China; ${ }^{14}$ Department of Intensive Care Unit, Beijing Haidian Hospital, Beijing, China; ${ }^{15}$ Department of Intensive Care Unit, Beijing Coal Group General Hospital, Beijing, China; ${ }^{16}$ Department of Intensive Care Unit, Peking Union Medical College Hospital, Beijing, China; ${ }^{17}$ Department of Intensive Care Unit, Fifth Medical Center of the PLA General Hospital, Beijing, China; ${ }^{18}$ Department of Intensive Care Unit, Beijing Chest Hospital, Capital Medical University, Beijing, China

"These authors contributed equally to this work.

Correspondence to: Donghao Wang. Department of Intensive Care Unit, Tianjin Medical University Cancer Institute \& Hospital, Tianjin, China. Email: donghaow@tom.com; Yun Long. Department of Intensive Care Unit, Peking Union Medical College Hospital, Beijing, China. Email: ly_icu@aliyun.com; Xuezhong Xing. Department of Intensive Care Unit, National Cancer Center/National Clinical Research Center for Cancer/ Cancer Hospital, Chinese Academy of Medical Sciences and Peking Union Medical College, Beijing, China. Email: xingxuezhong@cicams.ac.cn.
\end{abstract}

Submitted Apr 12, 2021. Accepted for publication Aug 11, 2021.

doi: 10.21037/apm-21-870

View this article at: https://dx.doi.org/10.21037/apm-21-870

\section{Introduction}

According to the guidelines of Chinese Society of Critical Care Medicine, critically ill patients who fulfill the following criteria should be admitted to intensive care unit (ICU) for intensive care (1). First, acute, reversible and life-threating organ or system failure patients should be admitted to ICU, who may recover after a short period of intensive care. Second, high-risk patients with potentially life-threating diseases may benefit from intensive care monitoring and effective treatment. Third, acute on chronic organ or system failure patients may recover to original or near original state after intensive care monitoring and effective treatment. Four, other critically ill patients who may benefit from intensive care monitoring and effective treatment. However, patients with chronic wasting disease or terminal state cancer will not benefit from intensive care monitoring, and should not admitted to ICU.

With the development of surgical procedures, radiotherapy, target therapy and immunotherapy, the survival rate of patients with advanced stage cancer has improved greatly (2). Cancer patients who developed complications such as sepsis, acute kidney injury or acute respiratory failure after neoadjuvant therapy may

$\wedge$ ORCID: 0000-0003-3441-305X. 
have similar short-term or improved long-term survival after intensive monitoring and treatment compared with patients with no neoadjuvant therapy (3). Rescue anticancer treatment may alleviate organ failure in some hematological malignancies who present with acute kidney injury or acute respiratory failure. Most importantly, advanced staged cancer does not equal to terminally ill diseases. Currently, only German Society of Hematology and Medical Oncology (DGHO), Austrian Society of Hematology and Oncology (OeGHO), German Society for Medical Intensive Care Medicine and Emergency Medicine (DGIIN), and Austrian Society of Medical and General Intensive Care and Emergency Medicine (ÖGIAIN) jointly issued consensus statement on cancer patients requiring intensive care support (4). It mainly focuses on patients who received non-operative therapies. A recent meta-analysis including nine trials showed no significant differences in postoperative mortality and complications rate between goal directed therapy group and control group in cancer patients undergoing high-risk surgery, but a reduced hospital length of stay was seen in goal directed therapy group (5). However, there are no consensuses or guidelines regarding perioperative intensive care and/or management of critically ill cancer patients. Therefore, we organized experts of some medical centers in China to draft consensus which focused on common important clinical problems in perioperative critically ill cancer patients, which provide reference for clinical intensivists and policymakers.

\section{Methods}

Details about the methods can be found in the Appendix 1 . The quality of evidence was graded according to GRADE system as followed (6). High quality, further research is very unlikely to change our confidence in the estimate of effect. Moderate quality, further research is likely to have an important impact on our confidence in the estimate of effect and may change the estimate. Low quality, further research is very likely to have an important impact on our confidence in the estimate of effect and is likely to change the estimate. Very low quality, any estimate of effect is very uncertain. The strength of recommendations is classified as strong if more than or equal to $1 / 2$ experts agree with the statement. Recommendation is classified as weak if less than $1 / 2$ experts agree with the statement.

The consensus was first prepared by two members (Wang HJ and Xing XZ) using search strategies in the Supplement, and twenty-one consensuses were formed.
Then these consensuses were sent to thirty experts of Critical Care Medicine Committee of Beijing Association of Oncology (CCMBAO) by electronic questionnaire and were graded. Finally, twenty-four experts responded. We present the following article in accordance with the RIGHT reporting checklist (available at https://dx.doi. org/10.21037/apm-21-870).

\section{Results}

\section{Question 1. Which kind of cancer patients may benefit from postoperative intensive care monitoring?}

Traditional definition of high-risk cancer patients is limited to patients with American Society of Anesthesiology (ASA) grade 3-4 or patients who underwent thoracic, abdominal or pelvic procedures (7-9). Experts from Massachusetts General Hospital proposed a new high-risk definition which includes twenty-three pre- and postoperative variables (10). In their study, patients who were rated as high propensity admission to ICU had decreased length of stay and costs after intensive care monitoring in ICU. On the contrary, patients who were rated as low propensity admission to ICU had increased length of stay and costs after intensive care monitoring in ICU, therefor these patients may return to surgical ward directly. However, external validation of the definition was not carried out owing to calculation method was not open.

(I) Consensus 1.1: the definition of high-risk patients refers to patients with ASA grade 3-4 who underwent surgical procedures or patients who underwent thoracic, abdominal or pelvic procedures (moderate quality, strong recommendation).

Traditional, high-risk surgical cancer patients including patients with ASA grade 3-4 and patients who underwent complex procedures may benefit from intensive care with reduced complications and mortality (7-9). Recently, several meta-analyses demonstrated no survival benefit for highrisk cancer patients after goal directed therapy in ICU $(5,9,11)$. However, for selected high-risk cancer patients, shortened hospital length of stay and reduced hospital cost were still found after goal directed therapy (10).

(II) Consensus 1.2: selected high-risk cancer patients may benefit from goal directed therapy with shortened hospital length of stay and reduced hospital cost (moderate quality, strong recommendation). 
Table 1 Surgical Apgar score

\begin{tabular}{|c|c|c|c|c|c|}
\hline Variables & 0 point & 1 point & 2 points & 3 points & 4 points \\
\hline Lowest mean arterial pressure $(\mathrm{mmHg})$ & $<40$ & $40-54$ & $55-69$ & $\geq 70$ & - \\
\hline Lowest heart rate (bpm) & $>85$ & $76-85$ & $66-75$ & $56-65$ & $\leq 55$ \\
\hline
\end{tabular}

Modified from reference (12).

Surgical Apgar score (SAS) was proposed in 2007, which includes three variables, estimated amount of blood loss, lowest mean arterial pressure and lowest heart rate. Zero to four points were assigned for each variable (Table 1) (12). SAS is calculated as sum of three variables and is predictive of postoperative death rate. The lower the score, the higher the mortality rate, and vice versa. SAS has been tested in various surgeries with satisfactory results. Calculation of SAS is simple, and it does not require biochemical workups, acute or chronic disease category, clinical assessment, or depend on the nature of the surgery (elective, urgent, emergency) (13). Therefore, SAS is a simple and useful triage tool for anesthesiologist, surgeons and intensivists.

(III) Consensus 1.3: SAS may serve as a new triage tool in high-risk patients (moderate quality, strong recommendation).

Calvo-Vecino et al. conducted a randomized controlled trial in 2018 which included 450 low-moderate risk surgical patients (14). They found that compared with control group, patients who received esophageal Doppler monitorguided goal-directed haemodynamic therapy had decreased complication rate $(8.6 \%$ vs. $16.6 \%, \mathrm{P}=0.018)$. However, there was no significant difference in mortality between two groups. The limitation of the study lies in small sample, and large study is needed to validate the conclusion.

(IV) Consensus 1.4: low-moderate risk surgical patients may benefit from esophageal Doppler monitor-guided goaldirected haemodynamic therapy with decreased complication rate (moderate quality, strong recommendation).

Perioperative goal-directed therapies consist of cardiac output-guided hemodynamic therapy, or esophageal Doppler monitor-guided goal-directed haemodynamic therapy, or cardiac index (CI)-guided goal-directed hemodynamic therapy, et al. $(5,9,11,14)$, which make it difficult to compare the results of different studies. Recently, a network meta-analysis demonstrated that goal-directed hemodynamic therapy aimed at intravascular volume, stroke volume, and cardiac output optimization are likely most effective (15). However, there is no universally accepted definition of goal-directed hemodynamic therapy. Therefore, unification of goal-directed therapy may help compare and popularize the results of the study.

(V) Consensus 1.5: inconsistency of perioperative goal-directed therapy deserves further high-quality studies with uniform definition (moderate quality, strong recommendation).

\section{Question 2. Does neoadjuvant therapy increase the mortality rate of critically ill cancer patients?}

Although neoadjuvant therapy may improve long-term survival of some resectable solid tumors, it increased the incidence of pulmonary embolism, decreased cardiopulmonary reserve $(16,17)$. It may also affect shortterm outcome of critically ill cancer patients. In a recently retrospective study involving 963 surgical patients, after propensity score matching, neoadjuvant therapy does not adversely affect the short-term outcome of critically ill cancer patients (3). In multivariable analysis using the same database after adjusting for age, gender and tumor staging, neoadjuvant therapy still does not adversely affect the shortterm outcome of critically ill cancer patients.

(I) Consensus 2.1: neoadjuvant therapy does not increase hospital mortality of critically ill cancer patients (moderate quality, strong recommendation).

\section{Question 3. Can extracorporeal membrane oxygenation (ECMO) be used in the treatment of perioperative severe/ refractory acute respiratory distress syndrome (ARDS) cancer patients?}

According to a meta-analysis in 2019 involving 429 severe/ refractory ARDS patients, usage of ECMO was associated with reduced 60-day mortality compared with controls, and 60 -day mortality was $34 \%$ and $47 \%$ in ECMO and control group respectively (18). In an international multicenter retrospective study, 6-month mortality was $34 \%$ and $66 \%$ 
for severe ARDS patients without or with immunodeficiency who received ECMO (19). Subgroup analysis demonstrated that hematological malignancies had the lowest 6-month survival compared with other immunodeficiency severe ARDS patients and patients with solid tumor $(\mathrm{P}=0.02)$. In another study including 68 nonhematologic malignancy patients with respiratory or cardiac failure who received ECMO as salvage therapy despite conventional therapy, the 6-month survival was $26.5 \%$ (20).

(I) Consensus 3.1: highly selected critically ill cancer patients with severe/refractory ARDS may benefit from ECMO therapy (moderate quality, strong recommendation).

There are case reports that ECMO therapy serves as a bridge therapy for refractory respiratory failure after esophagectomy $(21,22)$. Besides, ECMO has been successfully used for perioperative support in thoracic and airway surgery with a low perioperative mortality rate (23).

(II) Consensus 3.2: ECMO may be used as a bridge therapy for perioperative refractory respiratory failure and perioperative support for high-risk surgery (high quality, strong recommendation).

\section{Question 4. How is the prognosis of critically ill cancer patients with acute kidney injury?}

Results from South Korea demonstrated that the prevalence of acute kidney injury (AKI) was 33.8\% in 67,896 cancer patients according to risk, injury, failure, loss of kidney function, and end-stage kidney disease (RIFLE) criteria (24). Another study from China showed that there were 1,418 malignancy-related AKI in 7,604 AKI patients according to KDIGO criteria (25). Both patient-specific and cancer-related risk factors including age, co-morbid conditions, chemotherapy, surgery and radiotherapy increase the risk of AKI in cancer patients (26). Critically ill cancer patients with AKI had increased mortality compared patients without AKI (27).

(I) Consensus 4.1: many factors including surgery, chemotherapy, radiotherapy, and sepsis increase the risk of acute kidney injury in cancer patients. Acute kidney injury was associated with increased mortality in critically ill cancer patients (high quality, strong recommendation).

Several studies demonstrated that whether solid tumor or hematological malignancy patients, the prognosis of AKI was related to disease severity and AKI grading, but not cancer staging (28-31). Critically ill cancer patients with
AKI who received renal replacement therapy had similar short-term outcomes compared with AKI patients without cancer (29).

(II) Consensus 4.2: short-term outcome of cancer patients suffered from acute kidney injury was related to disease severity and acute kidney injury grading, but not cancer staging. Therefore, the indication of renal replacement therapy in cancer patients with acute kidney injury should not be different from non-cancer patients with acute kidney injury (moderate quality, strong recommendation).

\section{Question 5. Could ECLS be used in critically ill cancer patients with post-bepatectomy liver failure (PHLF)?}

PHLF comes from two aspects, primary or secondary causes. Primary PHLF refers to liver failure caused by small-for-size situation, coupled with intraoperative bleeding or not. Secondary PHLF refers to liver failure caused by late complications (32). According to definition and grading of International Study Group of Liver Surgery (ISGLS), PHLF was divided into three groups. Grading A PHLF refers to patients with abnormal laboratory parameters but require no change in the clinical management. Grading B PHLF is a state that patients have abnormal laboratory parameters, require albumin infusion, daily diuretics, or noninvasive ventilation. Grading C PHLF means patients not only have abnormal laboratory parameters, but require ICU admission and invasive treatment including vasoactive drugs, or hemodialysis, or intubation and mechanical ventilation, or ECLS, or rescue hepatectomy/liver transplantation (33). In the ISGLS study, the incidence of grading B and grading $\mathrm{C}$ was $8.1 \%$ and $2.2 \%$, with a mortality $12 \%$ and $54 \%$ respectively. In a Germany study which included 415 PHLF patients, the incidence of grading $\mathrm{B}$ and grading $\mathrm{C}$ was $5.7 \%$ and $1.6 \%$, with a mortality $12.5 \%$ and $85.7 \%$ respectively (34). In a China study which involving 1,683 patients, the incidence of grading $\mathrm{B}$ and grading $\mathrm{C}$ was $0 \%$ and $1.6 \%$, with a mortality $0.5 \%$ and $50 \%$ respectively (35).

(I) Consensus 5.1: the mortality of posthepatectomy liver failure was high although its incidence was low (high quality, strong recommendation).

There are no randomized controlled trials because of low incidence of PHLF. Treatment strategy of PHLF was based on the experiences of medical treatment of acute liver failure, which included elimination of possible etiology, 
supportive therapy of organ dysfunction, and treatment of postoperative reversible factors (36). Specific treatment measures consist of maintaining hemodynamic stability, blood purification for acute kidney injury or hepatic encephalopathy, prevention of gastrointestinal bleeding, treatment of postoperative infection. The outcome of ECLS was related to the etiology of PHLF. In a meta-analysis in 2020, of 34 PHLF patients who received ECLS, ninetyday mortality was $60 \%$ and $17 \%$ respectively for primary PHLF ( $\mathrm{n}=25)$ and secondary PHLF ( $\mathrm{n}=7)$ patients (32). Liver transplantation may be the only treatment measure for primary PHLF which was refractory to conventional measures. In a recent study, five-year and ten-year survival was $57 \%$ and $38 \%$ respectively for seven primary PHLF patients after liver transplantation (37).

(II) Consensus 5.2: specific treatment measures for posthepatectomy liver failure included elimination of possible etiology, supportive therapy of organ dysfunction. ECLS and liver transplantation may be an option for selected posthepatectomy liver failure patients (moderate quality, strong recommendation).

\section{Question 6. What is the clinical characteristic and prognosis of perioperative stress cardiomyopathy?}

In a national study in US, of 5,991,314 patients, patients with a prior intrathoracic/mediastinal malignancies and radiotherapy had increased risk of stress cardiomyopathy (38). Many anti-tumor agents were also associated with increased risk of stress cardiomyopathy in cancer patients (39). Patients who underwent major cancer surgeries were also at risk of developing stress cardiomyopathy (40). Compared with non-cancer patients who developed stress cardiomyopathy, cancer patients who diagnosed as stress cardiomyopathy had increased risk of respiratory insufficiency, prolonged hospital length of stay, slower recovery of cardiac function, and were more likely to develop cardiac arrest $(41,42)$.

(I) Consensus 6.1: anti-tumor therapies were associated increased risk of stress cardiomyopathy. Cancer patients who developed stress cardiomyopathy had worse outcome compared with patients without cancer (high quality, strong recommendation).

For stress cardiomyopathy patients with stable hemodynamics, treatment strategies were similar to cardiac failure patients with reduced heart function, which consists of usage of $\beta$-blocker, angiotensin conversion enzyme inhibitor or angiotensin II blocker, giving diuretics in fluid overload, avoiding inotropes in patients with left chamber outflow obstruction in case of obstruction aggravation (39). The annual recurrence rate of stress cardiomyopathy in patients who stop medical agents is $1.5 \%$, while $\beta$-blocker or angiotensin II blocker reduce the risk of recurrence (43). A retrospective study conducted in MD Anderson cancer center showed that of twenty-one stress cardiomyopathy patients who re-received anti-tumor systematic therapy, sixteen patients received treatment safely without recurrence of stress cardiomyopathy (44). However, there is also case report that stress cardiomyopathy patient developed cardiac arrest after re-received 5 -fluorouracil chemotherapy (45).

(II) Consensus 6.2: treatment strategies of stress cardiomyopathy were similar to cardiac failure patients. Cancer patients who had a history of stress cardiomyopathy should be assessed cautiously when re-receiving anti-tumor systematic therapy is needed (moderate quality, strong recommendation).

\section{Question 7. Does perioperative use of analgesic and sedation affect the outcome of cancer patients?}

Sedation is an important part of treatment of critically ill cancer patients. A follow-up study of randomized controlled trial demonstrated that low dose infusion of dexmedetomidine between the day during surgery and the first day of surgery was associated with increased two-year survival in cancer patients compared with controls, although no significant difference was found (46). The beneficial effect of dexmedetomidine lies in inhibition of inflammatory response and related tissue injury (47). However, a propensity score-matched retrospective study demonstrated that no association was found between intraoperative use of dexmedetomidine and 10-year survival in non-small cell lung cancer patients (48). In a large study included over 10,000 patients, increasing dose of propofol was associated with lower thirty-day postoperative complications, but not one-year mortality in solid tumor patients (49). In a metaanalysis including 7,866 cancer patients, the use of propofol was associated with improved survival compared with use of volatile anesthesia (50). However, in a recent randomized controlled study, combination use of paravertebral block and propofol was not associated with reduced recurrence rate in breast cancer after surgery compared with volatile 
anaesthesia and analgesic (opioids) (51).

(I) Consensus 7.1: sedation may improve short-term and longterm survival in perioperative cancer patients. However, further studies are needed to clarify the association between sedation and outcome of surgical cancer patients (moderate quality, strong recommendation).

In a prospective multicenter study including 1,381 postprocedural patients, $42 \%$ patients received pain assessment and $90 \%$ patients received opioids (52). In another study involving 151 patients receiving mechanical ventilation, the development of overt pain which was defined as a behavioral pain scale (BPS) of $>5$ was associated with increased thirtyday mortality and prolonged duration of mechanical ventilation (53). Study also showed that pain assessment and timely use of analgesia was associated shortened duration of mechanical ventilation (54). In a special article of American Society of Regional Anesthesia and Pain Medicine and the European Society of Regional Anaesthesia and Pain Therapy, there were two recommendations regarding analgestics and immune function. First, animal and human data demonstrated that morphine has negative effects on immune function. Second, non-opioid analgesia such as nonsteroidal agents do not comprised immune function (55).

(II) Consensus 7.2: pain assessment and analgesia were associated with improved short-term outcome in cancer patients. Further studies are needed to assess the association between analgestics and cancer relapse and recurrence (moderate quality, strong recommendation).

\section{Question 8. What is the role of adjuvant hemodialysis therapy in the treatment of postoperative severe intra- abdominal infection?}

According to a Chinese guideline for the diagnosis and management of intra-abdominal infection, severe intraabdominal infection was defined as intra-abdominal infection leads to sepsis, or patients suffers from intraabdominal infection with acute physiologic and chronic health evaluation (APACHE) II large than 10, or intraabdominal infection coupled with grading III or IV gastrointestinal injury (56). Therapeutic strategies of intra-abdominal infection consist of source control, antibiotic therapy and organ supportive therapy, nutritional therapy and adjuvant renal replacement therapy. A metaanalysis including ten studies involving 541 septic patients demonstrated that compared with controls, continuous blood purification increased 28-day survival rate and reduced duration of mechanical ventilation (57). A single center study in 2019 demonstrated that compared with controls, continuous renal replacement therapy reduced disease severity (APACHE II and sepsis-related organ failure assessment score) in severe intra-abdominal infection patients. Levels of inflammatory mediators dropped greatly in continuous renal replacement therapy group than controls. However, there was no significant difference in 28-day mortality between two groups (58).

(I) Consensus 8.1: adjuvant hemodialysis therapy may improve the severity of severe intra-abdominal patients (moderate quality, strong recommendation).

\section{Question 9. What is the role of metagenomic next- generation sequencing (mNGS) tests in the diagnosis and treatment of pulmonary infection after cancer surgery?}

Palacios et al. first using high-throughput sequencing discovered a new arenavirus in three patients who died of a febrile illness 4 to 6 weeks after receiving visceral-organ transplantation from a single donor (59). Currently, mNGS tests have been used in detecting virus, fungus, bacteria, parasite and other rare pathogens. However, it was not commonly used in clinical practice owning to its relative high cost (60). Multiple studies demonstrated that for suspected pulmonary infections, mNGS tests have a higher sensitivity than conventional measures in detecting sample from bronchoalveolar lavage fluid or sputum $(61,62)$. In addition, mNGS test is less affected by prior antibiotic exposure.

(I) Consensus 9.1: meagenomic next-generation sequencing has a higher sensitivity than conventional tests in detecting pulmonary infections (high quality, strong recommendation).

Grumaz et al. reported that 48 patients suffering septic shock received mNGS tests. After majority experts voting, $53 \%$ of patients $(n=24)$ received adjustment of antibiotic regimen, 38\% ( $\mathrm{n}=18)$ continued the original regimen (63). Of 24 patients who received antimicrobial therapy adjustment, experts recommended a de-escalation in $80 \%$ and escalation in $40 \%$ patients. In addition, patients who received antimicrobial therapy adjustment based on a combination of mNGS tests and experts' opinions had absolute increasement of 28 and 90 -day survival $13 \%$ and $14 \%$ respectively compared with patients who remained original antibiotic based on experts' opinions alone. However, there were only 3 
patients diagnosed as pulmonary infections. In Lin et al. study, there were 32 patients diagnosed as pulmonary infections. Of 20 patients who received antimicrobial therapy adjustment based on a combination of mNGS tests and experts' opinions, 14 patients $(70 \%)$ had symptoms improvement (64).

(II) Consensus 9.2: meagenomic next-generation sequencing tests have a positive role in the treatment decision making of pulmonary infection (moderate quality, strong recommendation).

\section{Question 10. What is the role of cerebral oximetry in the monitoring of critically ill cancer patients after brain surgery?}

Sekhon et al. prospectively assessed the relationship between regional saturation of oxygen $\left(\mathrm{rSO}_{2}\right)$ and mean arterial pressure (MAP) using near-infrared spectroscopy in cardiac arrest patients (65). They found that there is positive relationship between $\mathrm{rSO}_{2}$ and MAP when cerebral autoregulation is damaged. Rivera-Lara et al. demonstrated that $\mathrm{rSO}_{2}$ was a useful tool in determining the upper and lower limits of cerebral autoregulation in the case of acute neurologic injury whose cerebral autoregulation is dynamic, but not static (66). Hence $\mathrm{rSO}_{2}$ may serve as an alternative non-invasive tool in determining cerebral perfusion pressure, instead of invasive intracranial pressure measurement. Rivera-Lara et al. also found that cerebral oximetry index (COx), a derivative parameter calculated using the relation between $\mathrm{rSO}_{2}$ and MAP, is associated with in-hospital mortality, 6-month mortality rate, and 6-month severe disability in acutely comatose adults with neurological injury $(67,68)$.

(I) Consensus 10.1: $\mathrm{rSO}_{2}$ and its derivative parameter may aid in clinical treatment decision and predicting the prognosis of acutely comatose patients after surgery (moderate quality, strong recommendation).

In a meta-analysis including five randomized controlled studies involving 446 patients, Liu et al. found that intraoperative cerebral oxygen saturation monitoring and intervention effectively reduced the occurrence of postoperative delirium in patients undergoing non-cardiac surgery (69). In their study, the treatment cutoff was absolute value $50 \%$ of $\mathrm{rSO}_{2}$, or decreased by $20 \%$ from baseline level. Treatment measures included adjustment of mean arterial pressure, pressure of $\mathrm{CO}_{2}$, hemoglobin level and cardiac function. There was significant difference in postoperative delirium rate between treatment group and controlled group ( $25.2 \%$ versus $34.1 \%, \mathrm{P}=0.04$ ). However, owing to the lack of high-quality, there is no sufficient evidence to support the routine use of $\mathrm{rSO}_{2}$ measure to decrease mortality rate of patients who undergoing non-cardiac surgery (70).

(II) Consensus 10.2: goal-directed $\mathrm{rSO}_{2}$ monitoring during non-cardiac surgery may decrease postoperative delirium rate (moderate quality, strong recommendation).

\section{Future research}

In summary, we make some recommendations about critical care for perioperative critically ill cancer patients. However, there are some limitations in our consensuses. First, few recommendations are based on high qualities, while most recommendations are based on moderate qualities evidences. Second, consensus development group consisted of clinical intensivists oncologist without surgeons. Costeffective analysis was not conducted owing to the shortage of relevant literatures. Therefore, more trials regarding perioperative critical care for cancer patients are needed to provide high quality evidences for the updated consensus. For example, prospective studies are needed to determine whether usage of SAS as an ICU triage tool is a promising strategy in high-risk cancer patients in resource-limited hospitals, whether timing of renal replacement therapy impacts on the recovery of postoperative cancer patients with stage 2-3 AKI, whether adjuvant hemodialysis improve the short-term outcome of postoperative critically ill cancer patients with severe intra-abdominal infection.

\section{Acknowledgments}

Consensus panel: Haijun Wang, Hongzhi Wang, Wei Chen, Heling Zhao, Donghao Wang, Yun Long, Xuezhong Xing. External reviewers: Yuanyu Qian, Limin Shen, Shuangling Li, Jun Duan, Zhiqiang Wang, Keliang Cui, Quan Wang, Xiaoyan Xue, Xiyuan Li, Liwei Hua, Yingping Zhang, Yongshun Feng, Huaiwu He, Lei Li, Nan Zhang, Jun Dong, Weishuai Bian, Feiping Lu.

Funding: This work was supported by Beijing Hope Run Special Fund of Cancer Foundation of China (LC2020A22).

\section{Footnote}

Reporting Checklist: The authors have completed the 
RIGHT reporting checklist. Available at https://dx.doi. org/10.21037/apm-21-870

Conflicts of Interest: All authors have completed the ICMJE uniform disclosure form (available at https://dx.doi. org/10.21037/apm-21-870). The authors have no conflicts of interest to declare.

Ethical Statement: The authors are accountable for all aspects of the work in ensuring that questions related to the accuracy or integrity of any part of the work are appropriately investigated and resolved.

Open Access Statement: This is an Open Access article distributed in accordance with the Creative Commons Attribution-NonCommercial-NoDerivs 4.0 International License (CC BY-NC-ND 4.0), which permits the noncommercial replication and distribution of the article with the strict proviso that no changes or edits are made and the original work is properly cited (including links to both the formal publication through the relevant DOI and the license). See: https://creativecommons.org/licenses/by-nc-nd/4.0/.

\section{References}

1. Minister of Health of the People's Republic of China. Construction and management of Department of Critical Care Medicine (proposed edition). 2009. Available online: http://www.nhc.gov.cn/bgt/s9509/200902/45921149e2214 f89ae1b30e7ec1e19db.shtml

2. Darmon M, Bourmaud A, Georges Q, et al. Changes in critically ill cancer patients' short-term outcome over the last decades: results of systematic review with meta-analysis on individual data. Intensive Care Med 2019;45:977-87.

3. Xing XZ, Wang HJ, Qu SN, et al. Neoadjuvant therapy does not adversely affect the short-term outcome of critically ill cancer patients who underwent surgery. Transl Cancer Res 2020;9:294-9.

4. Kiehl MG, Beutel G, Böll B, et al. Consensus statement for cancer patients requiring intensive care support. Ann Hematol 2018;97:1271-82.

5. Gerent ARM, Almeida JP, Fominskiy E, et al. Effect of postoperative goal-directed therapy in cancer patients undergoing high-risk surgery: a randomized clinical trial and meta-analysis. Crit Care 2018;22:133.

6. Guyatt GH, Oxman AD, Vist GE, et al. GRADE: an emerging consensus on rating quality of evidence and strength of recommendations. BMJ 2008;336:924-6.
7. Ghaferi AA, Birkmeyer JD, Dimick JB. Variation in hospital mortality associated with inpatient surgery. $\mathrm{N}$ Engl J Med 2009;361:1368-75.

8. Gillies MA, Power GS, Harrison DA, et al. Regional variation in critical care provision and outcome after highrisk surgery. Intensive Care Med 2015;41:1809-16.

9. Sun Y, Chai F, Pan C, et al. Effect of perioperative goaldirected hemodynamic therapy on postoperative recovery following major abdominal surgery-a systematic review and meta-analysis of randomized controlled trials. Crit Care 2017;21:141.

10. Thevathasan T, Copeland CC, Long DR, et al. The Impact of Postoperative Intensive Care Unit Admission on Postoperative Hospital Length of Stay and Costs: A Prespecified Propensity-Matched Cohort Study. Anesth Analg 2019;129:753-61.

11. Pearse RM, Harrison DA, MacDonald N, et al. Effect of a perioperative, cardiac output-guided hemodynamic therapy algorithm on outcomes following major gastrointestinal surgery: a randomized clinical trial and systematic review. JAMA 2014;311:2181-90.

12. Gawande AA, Kwaan MR, Regenbogen SE, et al. An Apgar score for surgery. J Am Coll Surg 2007;204:201-8.

13. Lin YC, Chen YC, Yang CH, et al. Surgical Apgar score is strongly associated with postoperative ICU admission. Sci Rep 2021;11:115.

14. Calvo-Vecino JM, Ripollés-Melchor J, Mythen MG, et al. Effect of goal-directed haemodynamic therapy on postoperative complications in low-moderate risk surgical patients: a multicentre randomised controlled trial (FEDORA trial). Br J Anaesth 2018;120:734-44.

15. Zhao X, Tian L, Brackett A, et al. Classification and differential effectiveness of goal-directed hemodynamic therapies in surgical patients: A network meta-analysis of randomized controlled trials. J Crit Care 2021;61:152-61.

16. Sabra MJ, Smotherman C, Kraemer DF, et al. The effects of neoadjuvant therapy on morbidity and mortality of esophagectomy for esophageal cancer: American college of surgeons national surgical quality improvement program (ACS-NSQIP) 2005-2012. J Surg Oncol 2017;115:296-300.

17. Thomson IG, Wallen MP, Hall A, et al. Neoadjuvant therapy reduces cardiopulmunary function in patients undegoing oesophagectomy. Int J Surg 2018;53:86-92.

18. Munshi L, Walkey A, Goligher E, et al. Venovenous extracorporeal membrane oxygenation for acute respiratory distress syndrome: a systematic review and meta-analysis. Lancet Respir Med 2019;7:163-72. 
19. Schmidt M, Schellongowski P, Patroniti N, et al. SixMonth Outcome of Immunocompromised Patients with Severe Acute Respiratory Distress Syndrome Rescued by Extracorporeal Membrane Oxygenation. An International Multicenter Retrospective Study. Am J Respir Crit Care Med 2018;197:1297-307.

20. Park C, Ko UW, Ko RE, et al. Outcomes of extracorporeal membrane oxygenation in adults with active hematologic and nonhematologic malignancy. Artif Organs 2021;45:E236-46.

21. Zhong M, Tan L, Xue Z, et al. Extracorporeal membrane oxygenation as a bridge therapy for massive pulmonary embolism after esophagectomy. J Cardiothorac Vasc Anesth 2014;28:1018-20.

22. Mokashi S, Rajab TK, Lee LY, et al. Extracorporeal membrane oxygenation support after Ivor-Lewis esophagectomy for esophageal adenocarcinoma. Ann Thorac Surg 2014;97:1073-5.

23. Foong TW, Ramanathan K, Chan KKM, et al. Extracorporeal Membrane Oxygenation During Adult Noncardiac Surgery and Perioperative Emergencies: A Narrative Review. J Cardiothorac Vasc Anesth 2021;35:281-97.

24. Kang E, Park M, Park PG, et al. Acute kidney injury predicts all-cause mortality in patients with cancer. Cancer Med 2019;8:2740-50.

25. Jin J, Wang Y, Shen Q, et al. Acute kidney injury in cancer patients: A nationwide survey in China. Sci Rep 2019;9:3540.

26. Cosmai L, Porta C, Foramitti M, et al. Preventive strategies for acute kidney injury in cancer patients. Clin Kidney J 2021;14:70-83.

27. Lameire N, Vanholder R, Van Biesen W, et al. Acute kidney injury in critically ill cancer patients: an update. Crit Care 2016;20:209.

28. Darmon M, Vincent F, Canet E, et al. Acute kidney injury in critically ill patients with haematological malignancies: results of a multicentre cohort study from the Groupe de Recherche en Réanimation Respiratoire en OncoHématologie. Nephrol Dial Transplant 2015;30:2006-13.

29. Kemlin D, Biard L, Kerhuel L, et al. Acute kidney injury in critically ill patients with solid tumours. Nephrol Dial Transplant 2018;33:1997-2005.

30. Darmon M, Thiery G, Ciroldi M, et al. Should dialysis be offered to cancer patients with acute kidney injury? Intensive Care Med 2007;33:765-72.

31. Yuan ZN, Wang HJ, Qu SN, et al. The 28-day survive for critically ill cancer patients undergoing continuous renal replacement with postoperative acute kidney injure: a retrospective study of 86 cases. Transl Cancer Res 2020;9:6221-31.

32. Sparrelid E, Gilg S, van Gulik TM. Systematic review of MARS treatment in post-hepatectomy liver failure. HPB (Oxford) 2020;22:950-60.

33. Rahbari NN, Garden OJ, Padbury R, et al. Posthepatectomy liver failure: a definition and grading by the International Study Group of Liver Surgery (ISGLS). Surgery 2011;149:713-24.

34. Birgin E, Tesfazgi W, Knoth M, et al. Evaluation of the New ISGLS Definitions of Typical Posthepatectomy Complications. Scand J Surg 2019;108:130-6.

35. Zheng Y, Yang H, He L, et al. Reassessment of different criteria for diagnosing post-hepatectomy liver failure: a single-center study of 1683 hepatectomy. Oncotarget 2017;8:89269-77.

36. Søreide JA, Deshpande R. Post hepatectomy liver failure (PHLF) - Recent advances in prevention and clinical management. Eur J Surg Oncol 2021;47:216-24.

37. Thorsen T, Solheim JM, Labori KJ, et al. Liver transplantation as a lifesaving procedure for posthepatectomy liver failure and iatrogenic liver injuries. Langenbecks Arch Surg 2019;404:301-8.

38. Desai R, Desai A, Abbas SA, et al. National prevalence, trends and outcomes of takotsubo syndrome in hospitalizations with prior history of mediastinal/ intrathoracic cancer and radiation therapy. Int J Cardiol 2020;309:14-8.

39. Desai A, Noor A, Joshi S, et al. Takotsubo cardiomyopathy in cancer patients. Cardiooncology 2019;5:7.

40. Lechowicz-Glogowska E, Rojek A, Duszynska W. The Irreversible Neurogenic Stress Cardiomyopathy During Large Supratentorial Brain Tumor Resection. Neurocrit Care 2019;31:587-91.

41. Brunetti ND, Tarantino N, Guastafierro F, et al. Malignancies and outcome in Takotsubo syndrome: a meta-analysis study on cancer and stress cardiomyopathy. Heart Fail Rev 2019;24:481-8.

42. Zaghlol R, Kashyap K, Al-Shbool G, et al. Usefulness of Malignancy as a Predictor of WorseIn-Hospital Outcomes in Patients With Takotsubo Cardiomyopathy. Am J Cardiol 2019;123:995-1001.

43. Singh K, Carson K, Usmani Z, et al. Systematic review and meta-analysis of incidence and correlates of recurrence of takotsubo cardiomyopathy. Int J Cardiol 2014;174:696-701.

44. Weber J. Immune checkpoint proteins: a new therapeutic 
paradigm for cancer--preclinical background: CTLA-4 and PD-1 blockade. Semin Oncol 2010;37:430-9.

45. Basselin C, Fontanges T, Descotes J, et al. 5-Fluorouracilinduced Tako-Tsubo-like syndrome. Pharmacotherapy 2011;31:226.

46. Zhang DF, Su X, Meng ZT, et al. Impact of Dexmedetomidine on Long-term Outcomes After Noncardiac Surgery in Elderly: 3-Year Follow-up of a Randomized Controlled Trial. Ann Surg 2019;270:356-63.

47. Ueki M, Kawasaki T, Habe K, et al. The effects of dexmedetomidine on inflammatory mediators after cardiopulmonary bypass. Anaesthesia 2014;69:693-700.

48. Cata JP, Singh V, Lee BM, et al. Intraoperative use of dexmedetomidine is associated with decreased overall survival after lung cancer surgery. J Anaesthesiol Clin Pharmacol 2017;33:317-23.

49. Schaefer MS, Raub D, Xu X, et al. Association between propofol dose and 1-year mortality in patients with or without a diagnosis of solid cancer. Br J Anaesth 2020;124:271-80.

50. Yap A, Lopez-Olivo MA, Dubowitz J, et al. Anesthetic technique and cancer outcomes: a meta-analysis of total intravenous versus volatile anesthesia. Can J Anaesth 2019;66:546-61.

51. Sessler DI, Pei L, Huang Y, et al. Recurrence of breast cancer after regional or general anaesthesia: a randomised controlled trial. Lancet 2019;394:1807-15.

52. Payen JF, Chanques G, Mantz J, et al. Current practices in sedation and analgesia for mechanically ventilated critically ill patients: a prospective multicenter patient-based study. Anesthesiology 2007;106:687-95; quiz 891-2.

53. Yamashita A, Yamasaki M, Matsuyama H, et al. Risk factors and prognosis of pain events during mechanical ventilation: a retrospective study. J Intensive Care 2017;5:17.

54. Payen JF, Bosson JL, Chanques G, et al. Pain assessment is associated with decreased duration of mechanical ventilation in the intensive care unit: a post Hoc analysis of the DOLOREA study. Anesthesiology 2009;111:1308-16.

55. Missair A, Cata JP, Votta-Velis G, et al. Impact of perioperative pain management on cancer recurrence: an ASRA/ESRA special article. Reg Anesth Pain Med 2019;44:13-28.

56. Jian-An R. Chinese guideline for the diagnosis and management of intra-abdominal infection (2019 edition). Chin J Practical Surg 2020;40:1-16.

57. Wang LM, Cai YF, Dong JY, et al. Clinical effect of continuous blood purification in patients with sepsis: a meta-analysis. Chin J Crit Care Med (Electronic Edition) 2014;7:5-9.

58. Wu Y, Wang X, Wang L, et al. Early Continuous Renal Replacement Therapy (CRRT) in the Treatment of Severe Abdominal Infections-A Prospective Controlled Study of Single-center. J Med Research 2019;48:48-52.

59. Palacios G, Druce J, Du L, et al. A new arenavirus in a cluster of fatal transplant-associated diseases. N Engl J Med 2008;358:991-8.

60. Consensus Group Of Experts On Application Of Metagenomic Next Generation Sequencing In The Pathogen Diagnosis In Clinical Moderate And Severe Infections, Professional Committee Of Sepsis And Shock Chinese Research Hospital Association, Professional Committee Of Microbial Toxins Chinese Society For Microbiology, et al. Expert consensus for the application of metagenomic next generation sequencing in the pathogen diagnosis in clinical moderate and severe infections (first edition). Zhonghua Wei Zhong Bing Ji Jiu Yi Xue 2020;32:531-6.

61. Wang J, Han Y, Feng J. Metagenomic next-generation sequencing for mixed pulmonary infection diagnosis. BMC Pulm Med 2019;19:252.

62. Vincent JL, Brealey D, Libert N, et al. Rapid Diagnosis of Infection in the Critically Ill, a Multicenter Study of Molecular Detection in Bloodstream Infections, Pneumonia, and Sterile Site Infections. Crit Care Med 2015;43:2283-91.

63. Grumaz S, Grumaz C, Vainshtein Y, et al. Enhanced Performance of Next-Generation Sequencing Diagnostics Compared With Standard of Care Microbiological Diagnostics in Patients Suffering From Septic Shock. Crit Care Med 2019;47:e394-402.

64. Lin Q, Han LH, Ma YF, et al. Clinical value of NextGeneration Sequencing in the diagnosis and treatment of pneumonia. Shenzhen Journal of Integrated Traditional Chinese and Western Medicine 2020;30:66-7.

65. Sekhon MS, Smielewski P, Bhate TD, et al. Using the relationship between brain tissue regional saturation of oxygen and mean arterial pressure to determine the optimal mean arterial pressure in patients following cardiac arrest: A pilot proof-of-concept study. Resuscitation 2016;106:120-5.

66. Rivera-Lara L, Zorrilla-Vaca A, Healy RJ, et al. Determining the Upper and Lower Limits of Cerebral Autoregulation With Cerebral Oximetry Autoregulation Curves: A Case Series. Crit Care Med 2018;46:e473-7. 67. Rivera-Lara L, Geocadin R, Zorrilla-Vaca A, et al. Near- 
infrared Spectroscopy-derived Cerebral Autoregulation Indices Independently Predict Clinical Outcome in Acutely Ill Comatose Patients. J Neurosurg Anesthesiol 2020;32:234-41.

68. Lu W, Hou B, Wang D, et al. Application of Dynamic Monitoring of Regional Cerebral Oxygen Saturation for Severe Traumatic Brain Injury. Chin J Rehabilitation Theory Practice 2020;26:106-9.

69. Liu C, Xu Q, Sun G, et al. The effect of cerebral oxygen saturation monitoring during non-cardiac surgery on postoperative delirium: a Meta-analysis. J Youjiang Medical University Nationalities 2019;41:657-60,81.

70. Thiele RH, Shaw AD, Bartels K, et al. American Society for Enhanced Recovery and Perioperative Quality Initiative Joint Consensus Statement on the Role of Neuromonitoring in Perioperative Outcomes: Cerebral Near-Infrared Spectroscopy. Anesth Analg 2020;131:1444-55.

Cite this article as: Wang $\mathrm{H}$, Wang $\mathrm{H}$, Chen $\mathrm{W}$, Zhao $\mathrm{H}$, Qian Y, Shen L, Li S, Duan J, Wang Z, Cui K, Wang Q, Xue X, Li X, Hua L, Zhang Y, Feng Y, He H, Li L, Zhang N, Dong J, Bian W, Lu F, Wang D, Long Y, Xing X; on behalf of Critical Care Medicine Committee of Beijing Association of Oncology (CCMBAO). Expert consensus of perioperative intensive care and management of critically ill cancer patients (2021). Ann Palliat Med 2021;10(9):9331-9341. doi: 10.21037/apm-21-870 


\section{Supplementary}

\section{Appendix 1}

Using search strategies in Appendix 1, two authors (Wang H, Xing X) identified relevant studies in PubMed, Wanfang, from inception through April 2021 without language restrictions. We also manually screened the reference lists of all relevant articles to supplement the systematic search.

\section{Search strategy}

For question 1, high risk surgery AND goal directed therapy, were used.

surgical apgar score AND high risk, were used.

For question 2, neoadjuvant therapy AND critically ill AND cancer were used.

For question 3, extracorporeal membrane oxygenation AND acute respiratory distress syndrome AND immunocomprised were used.

extracorporeal membrane oxygenation AND surgery AND perioperative were used.

For question 4, acute kidney injury AND cancer AND prognosis were used.

For question 5, post-hepatectomy AND liver failure were used.

For question 6, perioperative stress cardiomyopathy were used.

For question 7, cancer AND (anesthesia OR sedation) AND (dexmedetomidine OR propofol) AND surgery were used.

For question 8, hemodialysis AND postoperative intra-abdominal infection were used.

For question 9, next-generation sequencing tests AND pulmonary infection were used.

For question 10, cerebral oximetry AND critically ill were used. 